\title{
A NOVEL IMPRESSION TECHNIQUE FOR FLABBY RIDGE MANAGEMENT IN EDENTULOUS PATIENTS - A CASE REPORT
}

\section{Dental Science}

Dr. Nidhi Beria*

Dr. Rajeev Singh

\section{Dr. Asha M.}

Rathod

Dr. Gaurang

Mistry

\section{Dr. Charusheela Sardar}

Post-graduate student, Department of Prosthodontics, D. Y. Patil University, School of Dentistry, Navi Mumbai, Maharashtra. *Corresponding Author

MDS Prosthodontics, Professor, Department of Prosthodontics, D. Y. Patil University, School of Dentistry, Navi Mumbai, Maharashtra.

MDS Prosthodontics, Professor, Department of Prosthodontics, D. Y. Patil University, School of Dentistry, Navi Mumbai, Maharashtra.

MDS Prosthodontics, Professor \& Head, Department of Prosthodontics, D. Y. Patil University, School of Dentistry, Navi Mumbai, Maharashtra.

MDS Prosthodontics, Professor, Department of Prosthodontics, D. Y. Patil University, School of Dentistry, Navi Mumbai, Maharashtra.

\begin{abstract}
Patients presenting with flabby or displaceable tissue continue to challenge even the most competent dental practitioners A fibrous or flabby ridge is a superficial area of mobile soft tissue affecting the maxillary or mandibular alveolar ridges. It can develop when hyperplastic soft tissue replaces the alveolar bone and is a common finding particularly in the upper anterior region of long-term denture wearers. Masticatory forces can displace this mobile denture-bearing tissue, leading to altered denture positioning and loss of peripheral seal. Under compression, the denture bearing area will exhibit localised mobility around the flabby tissue. Unless managed appropriately, such "flabby ridges" adversely affect the support, retention, and stability of complete dentures. Many impression techniques have been proposed to help overcome this difficulty Selective impression techniques have long been used in such cases, ensuring the displaceable tissue is recorded 'at rest'. Subsequently, the denture will maintain its peripheral seal during function This article presents a case report for prosthodontic rehabilitation of a patient with flabby ridge with a window tray impression technique, modified with dual trays.
\end{abstract}

\section{KEYWORDS}

Flabby ridge, impression, window technique, hypermobile tissue, mucostatic impression, complete denture.

\section{INTRODUCTION}

The success of complete denture prosthesis is often a reflection of its retention, stability and support offered during its usage in function. A definitive complete denture impression should record the entire functional denture-bearing, relieving and limiting areas to ensure maximum retention, stability and support for the prosthesis. Unfortunately, difficulties arise when the quality of denture-bearing areas are not ideal and demonstrate 'displaceable' or 'flabby ridges' in denture foundation. The available literatures indicate that the prevalence of flabby ridges occurs up to $24 \%$ of edentulous maxilla and in $5 \%$ of edentulous mandible. ${ }^{2}$ The flabby ridge, is a superficial area of mobile soft tissue, which can affect the retention of a maxillary or a mandibular removable denture.

Flabby tissue is a common finding in long-term denture wearers indicating a hyperplastic growth of mucosa that replaces alveolar bone. ${ }^{2}$ This hypermobile growth of maxillary and mandibular ridges affects the wearing of prosthesis by the patient. Due to the continued replacement of resorbing hard tissue with keratinised soft tissue, the dentures start to traumatise and harm the unsupported soft tissue underneath. This may lead to patient discomfort, uninhibited displacement of the dentures and imbalance during function. The illfitting dentures cause a constant trauma to the underlying tissues resulting in flabby ridges. ${ }^{3}$ Ellsworth Kelly in 1972 reported that mandibular anterior teeth cause trauma to maxillary anterior ridge as all occlusal forces are directed on to this area. This results in loss of bone from the anterior maxilla with subsequent fibrous tissue hyperplasia. The mucosa is highly movable and loosely attached to underlying periosteum of the bone. ${ }^{5}$ This flabbiness, is usually seen in anterior region of an edentulous mouth. ${ }^{6}$

So many therapies that are suggested in such cases include surgical excision of flabby mass, implant-supported dentures or conventional prosthesis without surgery. ${ }^{7-11}$ Implementation of treatment modality depends on medical health and requirement of the patient, extent of flabby tissue, financial burden on patient and skill of the prosthodontist. ${ }^{12}$

Management Of A Flabby Ridge Is Mainly By Three Approaches:

(1) Surgical removal of fibrous tissue prior to conventional prosthodontics,

(2) Implant retained prosthesis (i) fixed, (ii) removable,

(3) Conventional prosthodontics without surgical intervention.
A poor ridge is better than no ridge, which could be a sequel to surgical excision of the flabby tissues. There are various options available for the management of flabby ridge. These include surgical management of the hypertrophied soft tissue, implant supported removable or fixed dentures and conventional methods. Each technique has its advantages and shortcomings.

Window tray impression technique, initially introduced by Zarafulla Khan, has been modified and presented as an improved version, in this article. The purpose of this article is to describe an impression technique $^{16}$ for flabby ridges that makes use of impression plaster and low viscosity polyvinyl siloxane impression material, to record the flabby ridge and the well keratinised portion of the ridges, respectively, while incorporated into a dual-tray modified window tray.

\section{CASE-REPORT}

A 69 year old male patient, reported to the Department of Prosthodontics, D.Y. Patil University, School of Dentistry, Navi Mumbai, with a chief complaint of inability to chew food efficiently due to ill-fitting complete denture, which he had been using for the past 6 years. The dentures were completely worn occlusally, with total instability of the lower denture and stained throughout. On intraoral examination, it was found that the lower residual ridge was severely resorbed and the upper residual ridge was moderately resorbed and flabby in the premaxillary segment with a prominent incisive papilla and palatal rugae. The mucosa over the lower ridge was well keratinised and displaceable only in the floor of the mouth, which was placed higher than the lower ridge due to the severe resorption. (Fig. 1).

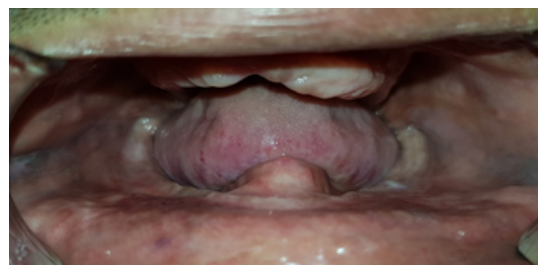

Figure 1 - Preoperative View

The primary impression for the edentulous mandible was made with 
modelling plastic impression compound and with irreversible hydrocolloid material for the edentulous maxilla, so as not to displace the flabby tissue situated anteriorly (Fig. 2). The extent of the displaceable tissue in the premaxillary region was marked, with an indelible pen, after adequate probing and examination, so as to provide adequate relief during the fabrication of custom tray.

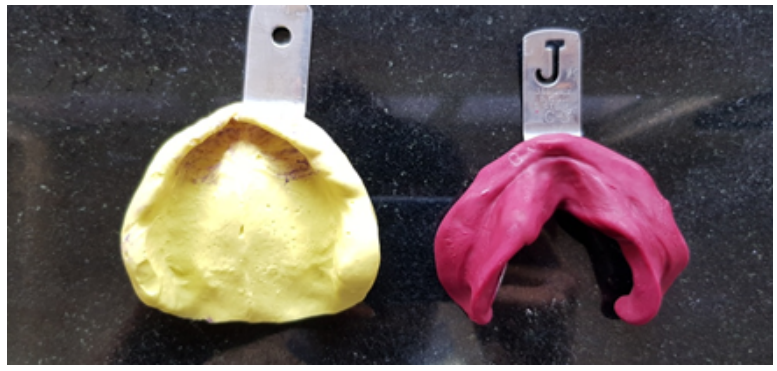

Figure 2 - Primary Impressions

The custom tray for the upper arch, was fabricated with a unique twotray technique; an inner tray and an outer pick-up tray. The inner tray was incorporated with a window cut out in the labial flange and two vertical retentive rods, in the posterior hard palate region, to orient and stabilise the outer tray onto the inner tray. The outer pickup tray was fabricated, with the two objectives : 1) To support the painted-on impression plaster material, in the window region of the inner tray, which was used to record the flabby region in a mucostatic state. 2) It was provided with holes in the region of the vertical rods of the inner tray, with a slightly active fit between the holes and the retentive rods, so as to facilitate improved grip on the inner tray, while retrieval of both the trays from the mouth, simultaneously. The inner tray was given adequate spacer to incorporate polyvinylsiloxane monophase material as the wash impression material. The outer pickup tray was incorporated with triple spacer that is upto $2.5 \mathrm{~mm}$ spacer thickness, and relief holes in the region of the flabby premaxilla, so as to achieve no displaceability of the mucosa during impression making. (Fig. 3) . The final impression was made in two stages - an A-silicone monophase material (medium body a-silicone; dentsply aquasil ultra monophase) wash impression for the unaffected well keratinised portion of the upper arch and an impression plaster wash, by the painton technique of the impression plaster for the flabby tissue portion. (Fig. 4).
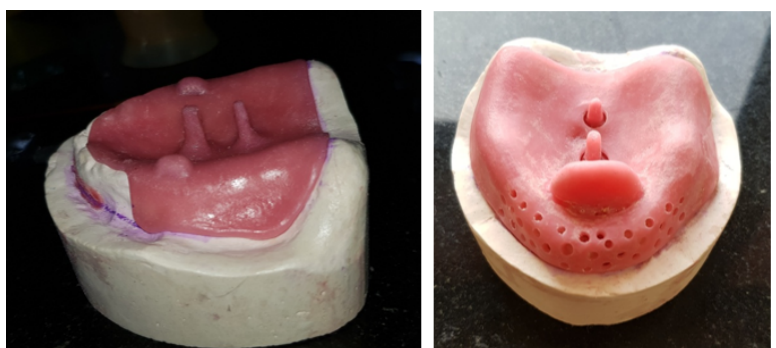

Figure 3 - Upper Arch Dual Custom Tray Fabrication

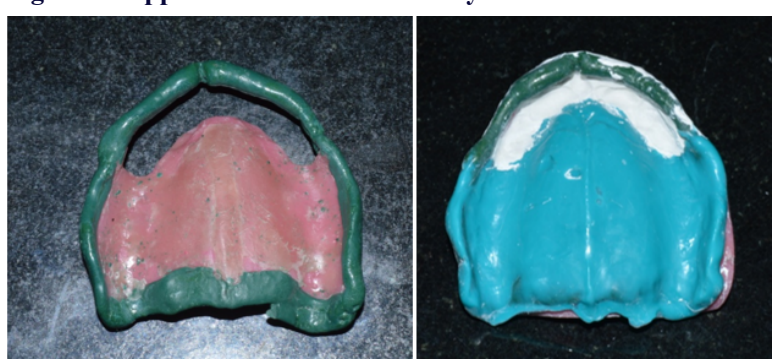

Figure 4 - Upper Arch Final Impression

The upper master model was poured with adequate application of separating medium on the flabby ridge impression area, to separate the impression plaster from the dental stone. The denture bases were fabricated in heat-cure acrylic resin, for better adaptability and lesser distortion. The patient's orientation jaw relation was recorded with a facebow transfer, the vertical and the horizontal jaw relations were recorded conventionally and the master models were mounted on a semi-adjustable articulator

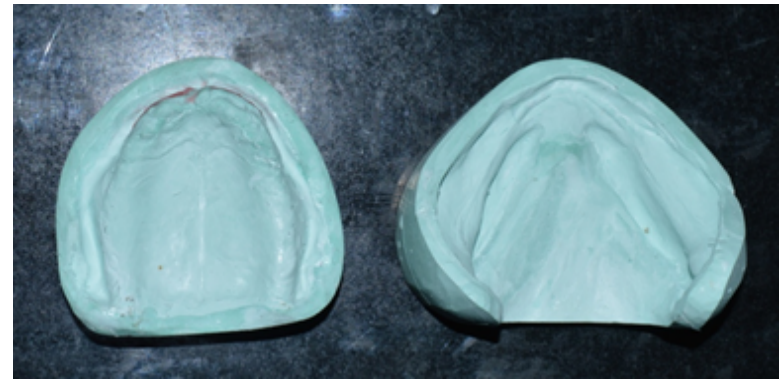

Figure 5-Master Models

The lower wax occlusal rim was removed and the patient's neutral zone was recorded on this denture base on a simulated rim of impression compound, relined with tissue conditioner on all the lingual and buccal surfaces. With all the functional movements performed for the patient, the neutral zone thus recorded on the thermoplastic impression compound material was transferred onto a putty matrix placed all around the impression compound rim. With the help of the putty matrix, the impression compound rim was then replaced with a conventional wax occlusal rim and the teeth were set according to the neutral zone and zero degree balanced occlusion principles

Once the waxed up trial dentures were ready, they were checked in the patients mouth for aesthetics, phonetics and occlusion.
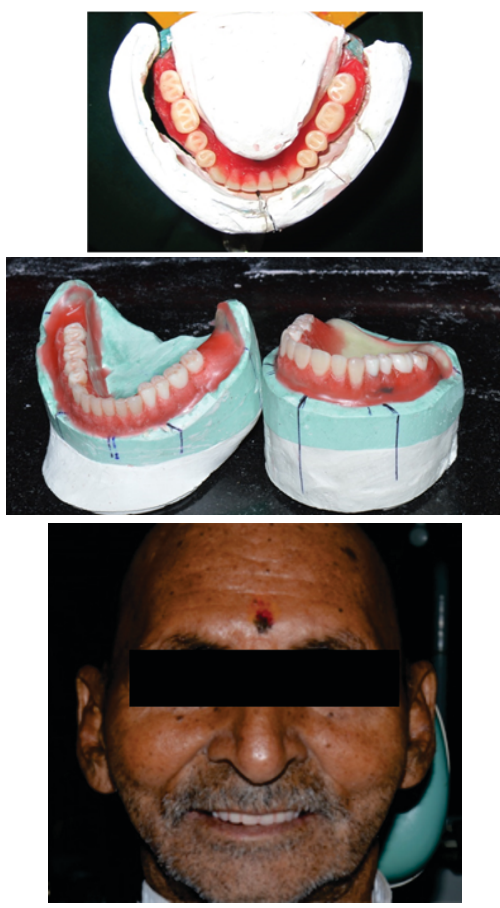

Figure 6 - Try-in of the dentures

\section{DISCUSSION}

Managing a patient with flabby maxillary ridge can be a challenging problem. Standard mucocompressive impression techniques are likely to result in an unretentive and unstable denture as the denture will be constructed on a model of the flabby tissue in a distorted state. Mucostatic techniques may not make the best use of the available tissue support and movement of the denture base relative to the support tissues may be a problem. The use of the principles of selective pressure or minimally displacive impression techniques, as was done with the window tray impression technique modified with dual trays, should help to overcome some of these limitations. ${ }^{13}$ With modified impression techniques, these ridges can be managed effectively without any additional clinical visits as compared to patients with normal edentulous ridges.

As a result of the resorbed mandibular ridge, the retention and stability for the lower denture was compromised. As a treatment modality for the excessively resorbed lower ridge, the patient's neutral zone was recorded with the objective of providing stability to the denture from 
the perioral musculature in a state of harmony with the patient's functional movements. ${ }^{18}$

An accurate impression of the edentulous ridge and functional sulcus is critical to the provision of a stable and retentive denture. Flabby ridges when recorded using a conventional method are compressed during impression. The elastic recoil of flabby fibrous soft tissue during function results in instability and loss of denture retention and dislodgement. ${ }^{19}$ Several impression techniques and methods have been described in the literature for recording flabby tissue during impression making. However, there is no evidence to support that one particular impression technique will provide a stable and retentive denture on flabby ridges as compared to others. ${ }^{20}$ This report presents a window tray impression technique modified with dual trays for the impression of anterior maxillary flabby ridge using the impression plaster paint-on technique.

Moreover, the window tray impression technique, modified with dual trays, allows for controlled application of low viscosity materials in addition to the minimal exertion of pressure to the flabby ridges due to the presence of properly sized vents.

Zero degree teeth were used in the fabrication of complete denture for this patient to minimize all horizontal or lateral forces, which may amplify due to the severely resorbed condition of the mandibular ridge, and thus dislodge or unstabilise the denture in the patient's mouth. ${ }^{20}$

\section{REFERENCES}

1. The British Society for the Study of Prosthetic Dentistry. Guidelines in Prosthetic and Implant Dentistry. London: Quintessence; 1996.

2. Xie Q, Närhi TO, Nevalainen JM, Wolf J, Ainamo A. Oral status and prosthetic factors related to residual ridge resorption in elderly subjects. Acta Odontol Scand 1997;55:306-13

3. Carlsson GE. Clinical morbidity and sequelae of treatment with complete dentures. J Prosthet Dent 1998; 79:17-23

4. E. Kelly, "Changes caused by a mandibular removable partial denture opposing a maxillary complete denture," The Journal of Prosthetic Dentistry, vol. 27, no. 2, pp. $140-150,1972$

5. Crawford RW, Walmsley AD. A review of prosthodontic management of fibrous ridges. BrDent J 2005;199:715-9.

6. McCord JF, Grant AA. Impression making. Br Dent J 2000;188:484-92

7. Lynch CD, Allen PF. Management of the flabby ridge: Using contemporary materials to solve an old problem. Br Dent J 2006;200:258-61.

8. Pai UY, Reddy VS, Hosi RN. A single step impression technique of flabby ridges using monophase polyvinylsiloxane material: A case report. Case Rep Dent 2014;2014:1045 41 .

9. Labban N. Management of the flabby ridge using a modified window technique and polyvinylsiloxane impression material. Saudi Dent J 2018;30:89.

10. Sankeshwari B. Impression techniques for management of flabby ridges in complete dentures-Areview. IDA J 2013;46:87-93

1. Bansal R, Kumar M, Garg R, Saini R, Kaushala S. Prosthodontic rehabilitation of patient with flabby ridges with different impression techniques. Indian J Dent 2014;5:110-3

12. P. F. Allen and S. McCarthy, Complete Dentures from Planning to Problem Solving, Quintessence Publishing, London, UK, 2003.

13 G. E. Carlsson, "Clinical morbidity and sequelae of treatment with complete dentures," Journal of Prosthetic Dentistry, vol. 79, no. 1, pp. 17-23, 1998

14 Q. Xie, T. O. Närhi, J. M. Nevalainen, J. Wolf, and A. Ainamo, "Oral status and prosthetic factors related to residual ridge resorption in elderly subjects," Acta Odontologica Scandinavica, vol. 55, no. 5, pp. 306-313, 1997.

15. B. C. Magnusson, H. Engström, and K.-E. Kahnberg, "Metaplastic formation of bone and chondroid in flabby ridges," British Journal of Oral and Maxillofacial Surgery, vol. 24, no. 4,pp. 300-305, 1986 .

16. R. W. I. Crawford and A. D. Walmsley, "A review of prosthodontic management of fibrous ridges," British Dental Journal, vol. 199, pp. 715-7719, 2006.

17. K. P. Liddelow, "The prosthetic treatment of the elderly," British Dental Journal, vol. 117 , no. 5 , pp. $307-315,1964$

18. J. Osborne, "Two impression methods for mobile fibrous ridges," British Dental Journal, vol. 117 , no. 6,pp. 392-394, 1964

19. R. M. Watson, "Impression technique for maxillary fibrous ridge," British Dental Journal, vol. 128, no. 11, p. 552, 1970

20. C. D. Lynch and P. F. Allen, "Management of the flabby ridge: using contemporary materials to solve an old problem,"British Dental Journal, vol. 200, no. 5, pp. 258-261,2006

21. Dhumal P, Singh R, Mistry G, Rathod A, Full mouth rehabilitation of a patient with mutilated dentition. J Dent Spec 2019;7(2):122-126

22. Surbhi Jain et al., 2019, Lingualized Occlusion- A Case Report. Int J Recent Sci Res. 10(09), pp. 34784-34787

23. Dr. Amruta AG, Dr. Rajeev S, Dr. Gaurang M and Dr. Omkar S. Tooth supported overdenture: A case report. International Journal of Applied Dental Sciences. 2019; 5(3): 299-302. 